\title{
On Kinematic Contributions to Form Factors.
}

\author{
I. RESAICK \\ Brookhaven National Laboratory - Upton N. Y. \\ (Nuovo Cimento, 39, $641(1965))$
} read

At the top of page 643 , in the first paragraph, the equation and sentence should

$$
\frac{2 l+1}{2} \int_{-1}^{1} \mathrm{~d}\left(\cos \theta_{s}\right) P_{l}\left(\cos \theta_{s}\right) P_{l}\left(\cos \bar{\theta}_{s}\right) .
$$

Now $\cos \vec{\theta}_{s}$ and $\cos \theta_{s}$ are related by $\cos \vec{\theta}_{s}=\alpha \cos \theta_{s}+\beta$, with $\beta \ldots$.

In eq. (15), the expression inside the integral should be given by

$$
\ldots \int_{m+\mu}^{\infty} \frac{\mathrm{d} \bar{W}}{\bar{W}-W} \frac{I_{\mathrm{X}}^{3+\mathrm{B}}\left(\bar{W}, \Delta^{2}\right) f_{\pi}^{\frac{1}{2}+\mathrm{B}}\left(\bar{W}, \mu^{2}\right)}{\bar{p} \cdot \bar{J}_{-} \bar{J}_{+}^{\prime}} .
$$

\section{Photoprotons from Mg.}

G. Manuzio, G. Ricco and M. Sanzone

Istituto di Fisica dell'Università - Genova

(Nuovo Cimento, 39, $1057(1965)$ )

The $31 \mathrm{MeV}$ photoproton yield $Y_{E_{\mathrm{p}}>3.5 \mathrm{MeV}}=10 \mathrm{~cm}^{2} / \mathrm{mole} \mathrm{MeV}$ should be replaced by $Y_{E_{\mathrm{p}}>3.5 \mathrm{MeV}}=10^{-4} \mathrm{~cm}^{2} /$ mole $\mathrm{MeV}$. 